\title{
La ética en la gerencia, vista a través del desarrollo organizacional
}

\author{
Díaz Barrios, Jazmin*
}

\section{Resumen}

El objetivo de este trabajo es ver como se relacionan la Ética, los Valores y la Gerencia, con la herramienta llamada Desarrollo Organizacional, a partir de una revisión bibliográfica, la cual dio como resultado que el punto de encuentro de la Ética y la Gerencia, es la responsabilidad social, y esa responsabilidad es posible si individuo y organización poseen valores humanísticos, los cuales pueden ser desarrollados aplicando DO en las organizaciones ya que éste puede llevar a las mismas a transitar el camino de la búsqueda de sus propios valores a través del autoconocimiento, para llegar a la autovaloración, que las llevará a la autoafirmación y por ende, al reencuentro con los principios éticos fundamentales de nuestra sociedad.

Palabras clave: Ética, valores, gerencia, desarrollo, organización.

Recibido: 99-03-15. Aceptado: 99-12-07

* Lic. Cs. Administrativas, Esp. Economia Empresarial, Cursando Doctorado Cs. Gerenciales, Docente de LUZ. Dirección Sector Zapara, Res. Zapara, Torre II Ph. Tif: 422187, cel: 014-6640845. E-mail: jdbconsult @ cantv.net. 


\section{Management Ethics as Seen From the Pointof View of Organizational Development}

\section{Abstract}

The objective of this research is to see how Ethics, Values and Management are interrelated with the tools of Organizational Development, all based on a bibliographical review. The result indicated that the encounter point for Ethics and Management is in the area of social responsibility, and that this responsibility is only carried out if the individual and the organization possess humanistic values. These values can be developed by applying $O D$ in organizations since this can guide them along the path of a personal and institutional value search by means of self-knowledge. This leads to self-valorization, which leads to self-affirmation, and finally to an encounter with the fundamental ethical principles of our society.

Key words: Ethic, values, management, development, organization.

\section{Introducción}

La tendencia de la gerencia hasta ahora, ha sido centrarse en la tecnología, la estructura, los sistemas y procedimientos, más que en los seres humanos que los llevan a cabo; esto ha llevado a que los principios éticos no jueguen un papel primordial en el quehacer gerencial, y los valores derivados de esa ética sean considerados, incluso, ejercicios retóricos. Sin embargo, la gerencia que se visualiza para el nuevo milenio, es una gerencia centrada en el hombre, en el ser humano como ente protagónico del proceso.

Los nuevos enfoques centrados en las personas, desarrollan a las organizaciones de una manera armónica, de forma tal que el crecimiento sea beneficioso para los intereses organizacionales y de todos los individuos que la conforman.
Esta es la base de la tesis del Desarroilo Organizacional (DO), el cual se enmarca en valores humanísticos que permiten volver y reencontrarse con los principios éticos fundamentales de nuestra sociedad, que en los últimos tiempos habian sido dejados de lado.

Cómo se relacionan la ética, los valores, la gerencia y esa herramienta llamada $\mathrm{DO}$, para desarrollar armónicamente organizaciones, es el objetivo fundamental que persigue este trabajo, el cual esta sustentado en una revisión bibliográfica.

\section{Etica}

Comencemos ubicando a la ética en su contexto filosófico, como una ciencia material, la cual se ocupa de los objetos empíricos, una disciplina práctica, 
que no se basta a si misma y requiere de la polis para su desarrollo. Esta disciplina se refiere a la acción humana y estudia la conducta moral del hombre en sociedad (Escobar,1992:43). En ese sentido, la Ética se ocupará en esclarecer, reflexionar, fundamentar esa experiencia humana que es la moral. Buscar su razón de ser, sus orígenes, el sentido de su evolución a través de los tiempos y las distintas culturas.

Etimológicamente, el vocablo ETICA proviene del griego ETHOS, lo cual a través de los tiempos ha significado: hábitat, morada, temperamento, carácter, hábitos, modo de ser, etc. La ética no es natural del hombre, es una conquista del mismo a través de su vida. Es un conjunto de hábitos de los cuales el hombre se apropia, modificando su naturaleza. Para Aristóteles, las virtudes éticas o morales, se generan por una repetición de actos o costumbres, por eso en su obra Etica nicomaquea dice que "una golondrina no hace verano, tampoco un acto virtuoso hace feliz y dichoso al hombre" (Escobar, 1992: 42).

Lo anterior se refiere, a que las costumbres éticas se logran con una vida sistemática de virtud y no por actos aislados de heroismo. Sin embargo, en este momento hay que decir que el concepto actual de Ética no es tan amplio como el sentido del vocablo antiguo, ya que hay muchas costumbres y hábitos que corresponden al Ethos pero que no tienen que ver con el objeto de estudio de la Ética como actualmente se la concibe, es decir con la Moral.

Como se apuntó anteriormente, la Moral, objeto de estudio de la Ética, es un fenómeno eminentemente social, puesto que rige o regula las actividades del hombre en sociedad, por lo tanto no tiene sentido fuera del ser humano (el único capaz de tener conductas, ya que el resto de los animales solo tiene comportamientos) ni tendría sentido en soledad ya que el ser humano es un SER CONy la conducta moral no se concibe fuera del contexto social.

Siendo que la moral esta constituida por una serie de reglas y normas que se presentan como obligatorias, valiosas $y$ orientadoras de la actividad humana y que la Ética reflexiona y estudia la moral, se ha tipificado la Ética como una ciencia normativa, una ciencia práctica, por tener como objeto la conducta humana. Pero lo anterior no significa que esta ciencia pueda servir de recetario de lo permitido o no en sociedad, no debe confundirse una ciencia con su objeto de estudio, por el contrario y como afirma G.E. Moore (1964:18-19) - "Los filósofos a díferencia de los moralistas, no se ocupan de establecer reglas para distinguir aquellos modos de obrar que casi siempre, o siempre, son justos, o aquellos otros que son injustos, ni siquiera de dar listas de las cosas buenas o malas, sino que se esfuerzan por contestar cuestiones mucho mas generales y fundamentales, como las siguientes: ¿Qué es lo que queremos decir de una acción cuando la calificamos de justa o injusta?, ¿Qué es lo que significa decir de un estado de cosas que es bueno o es malo?".

\section{Valores}

Podríamos intentar conceptualizar los Valores, en términos generales, como el convencimiento de que determinadas 
creencias son buenas $y$ deben usarse como criterios para la toma de decisiones; dicho de otra manera: podría decirse que son los motivos argüidos por los individuos $u$ organizaciones para explicar la forma en la cual se hacen las cosas (Stoner et al,1996:201). Se dice que los Valores están insertos en el esquema de la cultura del ser humano. Pero, ¿ de dónde vienen? ¿Adónde van? : Todo parte, de las creencias del individuo, de esos supuestos básicos, acuerdos, principios, credos, filosofía y dogmas, compartidos que constituyen la base del lado pensante de la cultura (Egan, 1996:77). Ese lado pensante de la cultura de una sociedad $u$ organización, se complementa con las ideas que la sociedad precia y valora y que por lo mismo le sirven de base para actuar, esos son los Valores.

Una vez que el individuo $u$ organización tiene establecídos esos valores, ¿cómo se llega a la acción? A través de las normas y las actitudes. Las normas van a ser la serie de reglas y reglamentos que limitan la actuación, son los debieras, los deberias, las haz, los no hagas, los estándares, las políticas, las leyes, los tabúes que gobieman la conducta en su conjunto. Y esas normas van a llevar al individuo a tener una determinada actitud hacia las situaciones. Una situación valorada como buena y con normas a favor, tendrá mayor propensión a ejecutarse, que una situación valorada como mala y con normas en contra. Esta actítud lleva a la ejecución de los patrones de conducta, no por obligación sino por el convencimiento del individuo, la organización o la sociedad de que son buenos y es gratificante de alguna manera el actuar según esa conducta. Es el lado actuante de la cultura.
Para Garcia \& Dolan (1997: 61-65), la palabra Valortiene tres dimensiones, a saber: Una dimensión ético-estratégica, una dimensión económica y una dimensión sicológica.

En la dimensión ético-estratégica, Milton Rokeach (citado por García Dolan, 1997: 63) define Valor como "Una convicción o creencia estable en el tiempo de que un determinado modo de conducta o finalidad existencial es personal o socialmente preferible a su modo opuesto de conducta o a su finalidad existencial contraria." Lo anterior quiere decir que el valor se aprende y se utiliza como estrategia para alcanzar ciertos fines en una forma en la cual sabemos que es apropiada y dará resultados. Podría expresarse como una actitud "moral" o "ética". Un disvalor por su parte, seria la anomia, es decir aquellas situaciones individuales, organizacionales o sociales donde no existen opciones de valor claramente definidas y por lo tanto se desconoce que tipo de actuación sería preferible o mejor vista por el colectivo, que otras. Vale decir, aquellas en las cuales no hay un principio ético establecido.

La segunda dimensión, es la dimensión económica, es decir la valía, la importancia, la significación que tienen las cosas o pueden llegar a tener según el valor agregado que vayan obteniendo en los distintos procesos o pasos por los cuales transite. Empresarialmente se habla del valor y lo relacionamos generalmente con la calidad y productividad de los productos, los cuales son los que van a aumentar la eficiencia y por ende la rentabilidad (valor) de la organización. Esencialmente todas las técnicas modernas (calidad total, reingeniería, ISO 9.000) y 
las orientaciones organizacionales como Desarrollo organizacional o Administración por objetivos, buscan aumentar el valor económico de la organización.

Por último tenemos la dimensión sicológica, llamada la valentía, la cual se tipifica como la "Cualidad moral que mueve a acometer resueltamente grandes empresas y a afrontar $\sin$ miedo los peligros". En este sentido podríamos decir que ser ético es servaliente, porque en el mundo actual, actuar según las creencias y valores, operacionalizar aquello en lo que creemos, ser como diría Aristóteles: "virtuoso", amerita, VALOR.

Cuando hablamos de los valores éticos se nos ocurre la siguiente pregunta: ¿Debemos vivir "con" Ios VALORESo "para" los VALORES, son los VALORES el fin de la vida, o el medio para vivir como deseamos? Para García \& Dolan (1997), la respuesta es que debemos vivir con nuestros valores éticos y éstos van a representar los medios para vivir la vida que deseamos. Pero no todos nuestros valores éticos tendrán el mismo nivel y preponderancia, incluso no se actúa de igual forma ante ellos. En la vida se tienen unos objetivos existenciales que estarán representados por los valores finales y unos medios operativos para alcanzar los objetivos existenciales, los cuales estarán representados por los valores instrumentales. No podemos decir que exista una correspondencia biunivoca entre cada valor final y cada valor instrumental, sin embargo hay un peligro latente: un valor instrumental podría convertirse en un valor final, para un determinado individuo, cuando este le atribuye una importancia excesiva.
Cómo definimos nuestros objetivos existenciales (valores finales)? Esto va a responder a preguntas tales como: qué es para un individuo lo más importante en su vida? Qué es para él lo más importante para el mundo? Estos cuestionamientos darán una medida de hacia donde se va. Operacionalmente vamos a definir éstos como los valores personales y los valores ético-sociales.

La definición de los valores instrumentales, es decir los medios para lograr nuestros objetivos existenciales, va a estar representada por los caminos que se elijan para llegar; Cómo cree el individuo que hay que comportarse con quienes 10 rodean, qué cree que debe poseer para competir en la vida?. Estos van a ser sus valores ético-morales y sus valores de competencia. Cada uno de estos vaiores genera sentimientos diferentes tanto en el individuo como en las organizaciones. El no actuar coherentemente con nuestros valores éticos puede generar sentimientos de culpabilidad y remordimientos, mientras que el no actuar según nuestros valores de competencia podría entrañar una sensación de fracaso. Pero no siempre se generan esos sentimientos de culpabilidad y fracaso. Cuando los valores que tiene la sociedad o la organización no tienen que ver con el individuo, él no los valora, y por ende no le ocasiona sentimiento alguno, su no cumplimiento. Los valores "valen", cuando tienen algo que ver con la vida, antes no valen, antes son contenidos intelectuales acerca de los valores, pero no se relacionan con lo que pasa en nuestra vida real. La sociedad en la que nacemos y nos criamos o la organización donde nos desempeñamos predica unos valores y nosotros nos ve- 
mos impelidos a predicarlos también, pero como no los conocemos verdaderamente, no creemos en ellos y no vemos necesario practicarlos. No se puede valorar lo que no se conoce, los valores se aprenden por un ejercicio de reflexión propia, cuando forman parte de la historia personal o grupal. El autoconocimiento lleva a la autovaloración y ésta a la autoafirmación. Por eso tanto para el individuo como para la organización se debe propender al autoconocimiento, se deben crear mecanismos que lleven a él. Si logramos el autoconocimiento, el resto del proceso será autosostenido y tendremos coherencia entre lo que predicamos y lo que hacemos, percibiremos los valores como algo valioso y estimable y los sentiremos dentro de nuestra vida.

\section{La ética, los valores y la gerencia}

La Gerencia es el lugar de decisión donde se orienta la acción. Es el proceso de hacer que las actividades se cumpian con eficiencia y eficacia por medio de otras personas (Robbin \& Coulter, 1996:8). La función gerencial es direccional y directorial, es decir que señala la orientación a la meta y dirige el proceso para alcanzarla.

Al ser la Gerencia ese lugar de la decisión, apunta igualmente al lugar de la responsabilidad, puesto que cada decisión implica una deliberación, una preferencia, una valoración, para poder llegar a la decisión y posteriormente envuelve una acción coherente con la decisión. Todo este proceso lleva implicita la asunción de una responsabilidad y de la incer- tidumbre sobre los resultados de la decisión tomada.

Pero, ¿adónde apunta esta responsabilidad?, ¿Con quien tiene la Gerencia responsabilidades en la toma de decisiones? Este es el lugar donde coinciden la Ética y la Gerencia: en la Responsabilidad social.

No es posible eludir los aspectos de índole ética en las empresas al igual que no pueden omitirse en otras áreas de la vida. Gran parte de los aspectos éticos caen dentro de cuatro niveles que no son mutuamente excluyentes, por el contrario, cada nivel esta contenido en el siguiente: Sociedad, grupos (externos e internos), e individuo (Stoner et al, 1996: 117-118).

El primer nivel es el que atañe a la socledad. En este nivel, se plantean preguntas acerca de las instituciones básicas en una sociedad. Los problemas de segregación racial, por ejemplo, plantean preguntas éticas como: ¿Acaso es ético tener un sistema social en el cual a un grupo étnico se le nieguen los derechos fundamentales? Otro ejemplo de este nivel lo constituyen aquellos cuestionamientos que atañen al capitalismo. ¿Asigna el capitalismo los recursos en forma justa?, ¿Qué función debe cumplir el gobierno como entidad normativa y reguladora del mercado. ¿Deben tolerarse las desiguaidades marcadas en riqueza, posición social y poder? Por lo general estos planteamientos del nivel que atañe a la sociedad son objeto de un debate continuo entre las principales instituciones a las que competen. Como personas y como gerentes, cada uno de nosotros forma parte de ese debate y asume una 
cuota de responsabilidad en las decisiones que como empresa afecten a la sociedad como un todo o a un sector de ella. Cuando una empresa decide, por ejemplo, no limpiar sus desechos tóxicos, aun cuando contamine el ambiente, porque no hay un funcionamiento correcto de la Ley, esa empresa incurre en una responsabilidad social por sus decisiones, que plantean un dilema ético: Se debe destinar dinero a limpieza de desechos, reduciendo así las ganancias, pero preservando el ambiente, o debe ganarse lo máximo posible, aunque esto implique una destrucción en el mediano plazo del medio ambiente? La decisión dependerá de los valores de la empresa, de los valores del tomador de decisión y de lo valioso que sean para "él" cada uno.

El segundo nivel de planteamiento de índole ético atañe a los grupos de interés, accionistas, proveedores, consumidores, tenedores de bonos y demás. Aquí se plantean preguntas sobre como debe una empresa negociar con los grupos externos que son afectados por sus decisiones, y que trato deben tener los grupos de interés con las empresas.

Abunda este tipo de problemas en las organizaciones, la negociación interna es uno de ellos; otro es la obligación que corresponde a toda empresa de informar a sus clientes acerca de los peligros potenciales que entraña la utilización de sus productos. ¿Qué obligación tiene una empresa con sus proveedores, con la comunidad dentro de la cual funciona, con sus accionistas? ¿Cómo es que debe intentar esclarecer estos asuntos?. A este nivel los planteamientos tienen que ver con la política de las empresas y en un sentido más profundo a los valores de la misma. Los planteamientos que conciernen a los grupos de interés se refieren a las relaciones de una empresa con sus recursos claves. Todos los días cada gerente toma decisiones a este nivel y la forma en la cual deciden tiene que ver con aquello que consideran valioso para la organización, es decir con el esquema de los Valores.

El tercer nivel de índole moral puede denominarse como política interna, a este nivel, hacemos preguntas en cuanto a la naturaleza de las relaciones de una empresa con sus empleados, tanto en el nivel ejecutivo, como en el de obreros. ¿Qué tipo de contrato es justo? ¿Cuáles son las obligaciones recíprocas de los dirigentes y los empleados? ¿Qué derechos corresponden a los trabajadores?. Estas preguntas también influyen en el trabajo diario de un gerente: los despidos, incentivos, normas de trabajo, motivación y liderazgo, son todas categorías éticas que pertenecen al tercer nivel. En este nivel el gerente debe tener especial cuidado de no presentar incoherencias entre las acciones que tome con los empleados y los valores que publique la empresa como propios, ya que cada empleado será un juez conocedor de estos valores y creerá en ellos o no dependiendo de ese nivel de coherencia que detecte en sus superiores.

Por último entramos al nivel individual o personal. En este cuarto nivel el gerente se plantea preguntas acerca de cómo debe tratar a sus semejantes dentro de una corporación. ¿Debemos ser honestos con los demás sin importar las consecuencias? ¿Cuáles son nuestras obligaciones como seres humanos y a la vez como empleados que cumplen una 
función particular de trabajo, ante nuestros jefes, subordinados y colegas? Estos aspectos corresponden a las actividades cotidianas de la vida en cualquier organización. Detrás de ellos subyacen dos aspectos más extensos. ¿Tenemos derecho a tratar a la gente en función de nuestros fines personales? , ¿Podemos evitar10? De nuevo los valores son los únicos elementos que pueden salir en nuestro auxilio para guiarnos en el camino ético a seguir.

Cuando en el ámbito individual un gerente debe decidir, la Administración por Valores puede ser un gran aliado. Los tres actos de la vida planteados por Blanchard \& O'Connor, (1997: 22-23): Realizar, conectare integrar permite volver a la esencia de la vida y en cada momento hacer lo que se requiere: Hacer para ser, ser con y cambiar para ser.

El problema de la responsabilidad social, representa el valor ético-social de la organización, pero también se relaciona con el ético-moral y algunas veces puede ser contradictorio con los valores de competencia y personales que se haya definido la organización, pero a través del análisis de la Administración por valores (APV) la empresa puede encontrarse a sí misma y al bienestar social. Como plantea Blanchard \& O'Connor, (1997: 39) el proceso de la APV en sus tres fases requiere:

1. Aclarar nuestros valores, propósito y misión

2. Comunicar nuestros valores

3. Alinear nuestras prácticas diarias con nuestra misión y nuestros valores.

La utilización de ese proceso permite primero conocernos, saber que que- remos, quienes somos y quienes queremos ser, luego nos guía en el proceso de comunicar a empleados, clientes, proveedores y relacionados, incluso a la sociedad en general, cuales serán esos valores por los cuales se guiará. $Y$ en tercer lugar al alinear nuestros valores con la práctica diaria, simplificamos nuestras decisiones, ganamos el respeto de nuestros empleados, clientes, proveedores y sociedad en general y por encima de todo ganamos el respeto de nosotros mismos como tomadores de decisiones y rectores de organizaciones. Decisiones alineadas a los valores organizacionales, darán como resultado el objetivo ético de la función empresarial: Optimizar la toma de decisiones en función del bienestar neto de la sociedad, entendiéndose por bienestar neto el de la sociedad en general y el de la organización en particular.

Pero este 'bienestar neto de la sociedad' lleva a una pregunta que se hace Ortiz Ibar (1995:1-12): "¿Supone la ética un límite a las oportunidades de negocio?".

A esta pregunta el autor responde, que lo más característico de los principios morales no consiste en constituir una barrera para los negocios: más que a evitar algunos males se orienta a conseguir las mejores actuaciones posibles. Por otra parte comenta, que el mundo empresarial está lleno de intangibles, por tanto, las cosas no pueden juzgarse como verdaderas o convenientes solamente en función de que se las pueda cuantificar. El éxito, en cierto modo, también es un intangible. $Y$ para alcanzarlo no hace falta proponerse que otros no lo alcancen.

Sin embargo no todos los autores defienden esta tesis de la responsabili- 
dad social, Según Milton Friedman: "La empresa sólo tiene una responsabilidad social: usar su energia y sus recursos para actividades que aumenten sus utilidades, siempre y cuando respete las reglas del juego...[y] se dedique a una competencia franca y libre, sin engaños ni fraudes" (Citado por Stoner et al 1996: 111). El sostiene que el problema de la empresa es producir y ganar dinero y el resto es problema del gobierno $u$ otros entes.

\section{El Desarrollo Organizacional}

Comencemos por decir que entre los enfoques existentes para llevar a cabo cambio planeado en las organizaciones, se encuentra además de los entoques estructural, tecnológico y de ambiente físico, el enfoque orientado hacia las personas, el cual trata de modificar el comportamiento del empleado centrándose en sus habilidades, actitudes, percepciones y expectativas. (Robbins, 1996: 720)

El Desarrollo Organizacional (DO) viene a ser una de las herramientas para cambiar a las organizaciones basándose en las personas.

Para conceptualizarlo, podemos decir que: Es una técnica a largo plazo, con la cual se pretende mover a toda la organización a un nivel más alto de funcionamiento y mejorar, al mismo tiempo, el nivel de desempeño y satisfacción de la sus integrantes (Stoner, 1994:450). En otras palabras, trata de cambiar la forma en la cual trabajan juntas las personas para lograr los objetivos organizacionales y propios (Robbins \& Coulter, 1996:430).
Estos objetivos organizacionales y propios, se refieren al propósito de mejorar la efectividad de la organización (logro de las metas) y la salud de la organización (motivación, utilización e integración del recurso humano) (De Faria, 1995:30).

EIDO no es algo nuevo, se basa en muchas cosas que ya se encontraban en el caudal de las ciencias humanas, sociales y administrativas, pero se trata de un arte diferente. Lo nuevo del DO es que tiene un uso sistémico, integrado y flexible, toma en cuenta la parte inferior del iceberg organizacional (sistema humano/social) y lo integra con la parte superior (sistema técnico/administrativo), mediante una metodología y una dimensión diferentes: "Metas" y "Recursos" dentro del subsistema Económico, "Estructura" dentro del subsistema Administrativo y "Valores" dentro del subsistema humano/social. Compatibiliza eficacia y salud, maximizándolas e integrándolas (De Faria, 1995:39-41). En otras palabras, enfoca a la organización como un sistema sociotécnico.

Pero el DO se mueve dentro de ciertos valores e hipótesis acerca de las personas y las organizaciones, los cuales constituyen una parte integral del desarrollo organizacional, modelando las metas y los métodos del campo y distinguiendo al DO de otras estrategias de mejoramiento (French \& Bell, 1996:70).

Los valores del DO tienden a ser humanistas, optimistas y democráticos. Los valores humanistas proclaman la importancia del individuo. Respetan a la persona total, tratan a las personas con respeto y dignidad, asumen que todas poseen una valia intrínseca, consideran que todas las personas tienen el mismo 
potencial para el crecimiento y el desarroIlo. Los valores optimistas postulan que el ser humano es básicamente bueno, por lo tanto puede y quiere progresar y los valores democráticos aseveran el derecho de las personas a estar libres de abuso arbitrario del poder, un trato justo y equitativo para todos.

"Estos valores no surgen maduros de los individuos o sociedades, sino que se forman de las creencias colectivas de una era -el zeitgist, o espíritu de la época. A medida que se acumulaban estos ingredientes, fueron modelados en unos fundamentos de valores coherentes para la teoria y la práctica del desarrollo organizacional" (French\&Bell, 1996:71).

Lo anterior significa que como premisa básica el DO requiere que en la organización exista un espíritu inquisidor, investigación sistemática, análisis racional y los elementos intuitivos que posibilitan las innovaciones (valores de competencia). Además actitudes y valores basados en la libre expresión, influencia mutua, posibilidad de compartir el riesgo, responsabilidad, aceptación de las diferencias y el derecho a la privacidad. Pero se requiere descubrir habilidades para adquirir nueva información para conocerse a sí mismo, a los demás y sus relaciones con la sociedad. Tolerar muestras faltas y errores, probar nuevos medios de alcanzar las metas, incluso el cambio de la meta misma. Requiere un nivel de autoconocimiento. Por último requiere un enfoque sistémico en donde las personas hacen en conjunto, cosas de valor económico y social que no podrian o escogerian hacer por si solos, ya que el efecto sinérgico implica que el todo, es más que la suma de las partes, es decir que los individuos logran en equipo más y mejores resultados que la sumatoria de sus esfuerzos individuales (De Faria, 1995:39-43).

Sintetizando: Los valores que el DO requiere para su implantación son entre otros: Apertura, Apoyo, Autenticidad, Colaboración. Confrontación, Confianza, Enfoque de sistema abierto, Atención a las necesidades individuales y organizacionales, Honradez, Humanidad, Realización personal, Desarrollo, Trabajo en equipo, etc. Sin la existencia de estos valores, la aplicación de las técnicas de DO constituiría un mero maquillaje organizacional, pero no implicaría un cambio cultural que permitiera el desarrollo armónico que se pretende.

Lo anterior lleva a que previa a la aplicación del DO en una organización, se requiere, además de un diagnóstico de salud organizacional que nos evalúe el presente, $y$ un análisis de la cultura organizacional que nos aclare los supuestos, valores, normas y patrones de conducta de la organización, una alineación a los valores requeridos por el DO para garantizar o al menos aumentar el porcentaje de posibilidades de éxito de la aplicación del DO en las organizaciones. Un esfuerzo formal de la organización no tendria resultado si cada miembro en particular y todos en general no estuvieran alineados a ese cambio, a esas nuevas actitudes, percepciones y expectativas sobre su organización.

Manifiesta Burke (1988:14): "Casi nunca se puede lograr un cambio tratando los síntomas, y no habrá cambio organizacional si se dirige el esfuerzo hacia un intento de cambiar los miembros individuales, La dirección del cambio debe orientarse hacia la personalidad de la or- 
ganización, no hacia la personalidad del individuo".

\section{La ética gerencial, los valores y el desarrollo organizacional}

Stoner \& Freeman (1994:453) al plantear la Ética en la Administración, visualizan los valores del DO en tres niveles a saber: personal, grupal y organizacional:

En el ámbito personal, los agentes de cambio de DO consideran que los individuos tienen un deseo natural de desarrollo personal y crecimiento y que la mayoría de los empleados están esperanzados y son capaces de dar una contribución mayor a la organización. También creen que la satisfacción de las necesidades humanas y sus aspiraciones son un importante propósito de la vida organizacional. Por consiguiente, están interesados en la autorrealización individual y tratan de superar los factores organizacionales que desalientan el crecimiento personal. Cualquier medio no sirve para cualquier fin, y en este sentido la práctica del DO selecciona aquellos medios que puedan alinear los valores personales de individuos y organizaciones, que puedan desarrollar los valores de competencia que requiere la organización respetando los valores ético morales del individuo y de la organización misma.

En el ámbito de grupo, la práctica del DO supone que es importante para la gente ser aceptados por su grupo de trabajo y que la mayoría de los grupos no fomentan la exposición abierta de los sentimientos, Sin embargo, se cree que esconder los sentimientos tiene un efecto negativo sobre la buena voluntad del grupo, la capacidad para solucionar constructivamente los problemas, sobre la satisfacción laboral y el desempeño del trabajo, posiblemente porque lesione un valor ético moral del individuo como es la honestidad. Por consiguiente, alientan la conciencia y el desarrollo de los sentimientos como una parte integral de la vida de la organización.

En el ámbito organizacional, la práctica del DO supone que los vínculos entre los grupos de trabajo influyen en su eficiencia. Por ejemplo, habrá más coordinación entre los grupos de trabajo cuando todos los integrantes del grupo estén en posibilidad de interactuar y menos cuando la comunicación es entre los niveles gerenciales exclusivamente. Un segundo supuesto consiste en que las políticas y métodos de grupos grandes afectarán la forma en que interactuarán los grupos más pequeños. Por último, la práctica supone que las estrategias que se basan en que un grupo o departamento gane a expensas de otro no obtendrá éxito a largo plazo, posiblemente porque lesiona el valor ético-moral de la confianza y el respeto mutuo. Por el contrario, las organizaciones han de basarse en los enfoques aceptables para todos los grupos que intervienen. Otro valor importante es la equidad en el poder, dentro de una organización argumentando que es necesaria para la salud a largo plazo de la organización. En gran parte de los casos, la equidad en el poder significa incrementar la influencia de los subordinados. Sin embargo, en algunas organizaciones, como universidades, hospitales y alcaldías o gobiemos regionales, la gente de menor nivel puede detentar poder en exceso. 
La alineación a unos valores predeterminados puede constituirse en solución de esta corrupta situación.

La esencia del DO constituye en si misma una expresión de la necesidad que tiene la Gerencia de tener actitudes éticas en ella, es un encuentro con los valores a través de la búsqueda del ser humano. Los valores finales e instrumentales, personales, ético-sociales, ético-morales y de competencia que encontramos en el quehacer ético, están presentes en las premisas básicas del DO. Su aplicación en las organizaciones, constituiria un nuevo orden, donde la Gerencia estaría basada no en la explotación del hombre por el hombre, no en el aprovechamiento de la miseria humana, sino en el respeto del ser humano, en una verdadera búsqueda de la conexión e integración que hiciera una realidad el SER CON.

La aplicación del DO implica en las organizaciones una evaluación previa que la lleve al autoconocimiento, autovaloración y autoafirmación de su naturaleza y sus responsabilidades individuales, grupales y sociales.

\section{Conclusiones}

Los asuntos de índole Ético están presentes en las empresas, al igual que en otras áreas de la vida. La moral (objeto de estudio de la Ética) se presenta como obligatoria, valiosa y orientadora de la actividad humana y en ella están inmersos valores que guian al individuo en la toma de decisiones.

La Gerencia es el lugar de decisión de la organización, lo cual implica una valoración previa y una responsabilidad posterior. Es en esa responsabili- dad social donde coinciden la Gerencia y la Ética. Una en la ejecución, otra en la reflexión. Pero, la reflexión sobre esa responsabilidad, esta basada en los valores que individuo y organización posean y de la "valoración" que le den a esos valores. Esto implica un conocimiento de ellos, lo cual llevará a la afirmación de los mismos.

Ante el planteamiento de la nueva gerencia, centrada en el hombre, el desarrollo organizacional se constituye en una alternativa, con valores humanísticos, optimistas y democráticos, los cuales deben estar presentes para su implantación. Por lo tanto, la aplicación del DO en las organizaciones, puede llevar a las mismas a transitar el camino de la búsqueda de sus propios valores a través del autoconocimiento, para llegar a la autovaloración, que los llevará a la autoafirmación y por ende al reencuentro con los principios éticos fundamentales de nuestra sociedad, que en las últimas décadas habian sido dejados de lado.

\section{Bibliografía citada}

Blanchard K \& O'Connors M. (1997). Administración por valores, Editorial Norma, Colombia.

Burke, Warner (1988). Desarrollo organizacional. Punto de vista normativo, Addison-Wesley iberoamericana, México.

De Faria Mello, F. (1995). Desarrollo organizacional . Enfoque integral, Editorial Limusa, México.

Egan, Gerard (1996). El valor agregado de los empleados en las organizaciones, Prentice Hall Hispanoamericana S.A., México.

Escobar, Gustavo (1992). Etica, Mc Graw Hill México. 
French, W. \& Bell C. (1996). Desarrollo organizacional, Prentice Hall Hispanoamericana, México.

Garcia, S. \& Dolan (1997). La Dirección por valores, Mc. Graw Hill, Madrid.

Moore, G. (1964). Ética, Editora Nacional, México.

Ortiz lbar, José (1995). La hora de la Ética empresarial, Mc Graw Hill, Madrid.

Robbins, Stephen (1996). Comportamiento Organizacional. Teoria y Práctica, $\left(7^{\mathrm{a}}\right.$. edición) Prentice Hall, México.
Robbins $S$. \& Coulter M (1996). Administración, $5^{\text {a }}$ edición, Prentice Hall Hispanoamericana S.A., México.

Stoner J. \& Freeman E. (1994). Administración (5 Edición), Prentice Hall Hispanoamericana S.A., México.

Stoner J., Freeman R. \& Gilbert D. (1996). Administración (6 $6^{\mathrm{a}}$. Edición), Prentice Hall Hispanoamericana S.A., México. 